\title{
The Application of Nutrition Care Service on Patient with Liver Cirrhosis
}

\author{
Zhaolin Chen ${ }^{1}$, Jinglan Luo ${ }^{2,}$, Dongxiu Liang ${ }^{1}$, Guanhong Li $^{1}$, Danmei Weng ${ }^{1}$, Rui Xi $^{1}$ \\ ${ }^{1}$ Digestive Department, The First Affiliated Hospital of Jinan University, Guangzhou, China \\ ${ }^{2}$ Internal Medicine Department, The First Affiliated Hospital of Jinan University, Guangzhou, China
}

Email address:

2009gzjnu@163.com (Zhaolin Chen), tljl@jnu.edu.cn (Jinglan Luo), 286630107@qq.com (Dongxiu Liang),

191426127@qq.com (Guanhong Li), 1024338913 aqq.com (Danmei Weng), 1156974221@qq.com (Rui Xi)

${ }^{*}$ Corresponding author

\section{To cite this article:}

Zhaolin Chen, Jinglan Luo, Dongxiu Liang, Guanhong Li, Danmei Weng, Rui Xi. The Application of Nutrition Care Service on Patient with Liver Cirrhosis. Science Journal of Clinical Medicine. Vol. 9, No. 1, 2020, pp. 19-21. doi: 10.11648/j.sjcm.20200901.16

Received: March 20, 2020; Accepted: April 3, 2020; Published: April 13, 2020

\begin{abstract}
Objective: To assess the effect of nutrition care service on patient with liver cirrhosis. Methods: 72 participants was invited to join our study. They were diagnosed as liver cirrhosis from April 2018 to March 2020. We randomly assigned the participants to the control group $(n=37)$ and the intervention group $(n=37)$. For the intervention group and control group, on the one hand, control group patients received traditional nursing services. On another hand, the intervention group participants receive nutrition care service in the treatment process. We collect the data from test related equipment record and questionnaires of participants, the questionnaires include Self-Rating Anxiety Scale (SAS) and Self-rating depression scale (SDS). Another data from test related equipment record. Result: In nutritional status, the Albumin information shown intervention group have greater improvement after nursing that that of control group (from 59.24 \pm 5.07 to $49.16 \pm 2.01$ vs from $60.12 \pm 4.73$ to $57.72 \pm 2.33$ ). In complications status, control group and intervention group have similar result, they have few different in different complications. In mental health, intervention group have greater change in anxiety status than that of control group (from 58.6 \pm 7.4 to $47.2 \pm 7.1$ ). Conclusion: the nutrition care service provides great improvement to the treatment result. However, nutrition care service does not have a good effect on partial results.
\end{abstract}

Keywords: Nutrition Care, Nursing, Liver Cirrhosis

\section{Introduction}

Base on the report of World Health Organization, any kind of liver disease acts as a source for cirrhosis of the liver. Liver cirrhosis has become a significant health problem worldwide as it leads to 1.34 million deaths every year [1]. In liver cirrhosis, the patients' liver suffer chronic or long-lasting injury so that the liver ploddingly deteriorates, even the liver is unable to function normally in late period of cirrhosis [2]. In recent years, patients reported outcomes came to the fore, and several studies have demonstrated that health-related quality of life is impaired in patients with liver cirrhosis when compared to the healthy general population [3, 4]. Yang showed that cirrhosis was present in $94 \%$ of patients having hepatitis B and $97 \%$ of patients having hepatitis $C$ in the study. However, each year viral hepatitis B and hepatitis C affect 325 million people causing 1.4 million deaths all over the world [5]. Moreover, advanced cirrhosis is a fatal condition where the treatment options are very limited [6]. So it is essential to find out control measures of this chronic liver disease. For this purpose, optimal control theory has been applied to discuss the optimal control strategy to prevent hepatitis B and hence consequently to reduce the chronic cirrhosis transmission.

The dietetic discipline has developed the Nutrition Care Process (NCP) as an organized framework to standardize the provision of high-quality nutrition care $[7,8]$. The NCP is order to support communication about dietetics and increase acknowledgment of the value of dietetic care by other healthcare professionals [9]. The first Nutrition Care Process flowchart was published in 1994 to propose nutrition care indicators to the Joint Commission on Accreditation of Healthcare Organizations (now referred to as The Joint Commission) for patient care with paper-based workflows 
[10]. In 2017, United States government spend \$1.3 trillion into nutrition care costs. The nutrition nursing services address inadequate or excessive food intake, that include nutrient deficiencies or nutrient excesses related to fluid, vitamins and/or minerals, alterations in gastrointestinal function from the mouth to the colon, malnutrition, and food insecurity; and education and counseling for nutrition and health issues [11, 12]. Aim of our study is assess the effect of nutrition care service on patient with liver cirrhosis.

\section{Methods}

\subsection{Participants Enrollment and Survey Methods}

We invited 74 patients who were diagnosed as liver cirrhosis were investigated join into our study. Their diagnose time is from April 2018 to March 2020. We randomly assigned the participants to the control group $(n=37)$ and the intervention group $(n=37)$. For the intervention group and control group, we provide different nursing services to them. The control group patients received traditional nursing services in the treatment process, the traditional nursing services is the standard of nursing measure by the hospital. In intervention group, the participants receive nutrition care service in the treatment process. we collect the data from test related equipment record and questionnaires of participants, the data contains nutrition status, complications status, anxiety information and depression information. In addition, we collected data using the following questionnaires: Self-Rating Anxiety Scale (SAS) and Self-rating depression scale (SDS) [13, 14].

Their inclusion criteria were: (1) the patients were diagnosed as liver cirrhosis; (2) coagulation function was well; (3) Patients volunteered to participate in follow-up; Their withdraw criteria were: (1) patients had too many complications in treatment process; (2) The patient also had other stomach problems.

\subsection{Statistical Analysis}

Our data analyzer performed the statistical analysis by SPSS 22.0. The $\mathrm{P}$ value, t-test and chi-square test were associated with collection result were analyzed. Besides, the mean standard deviation for statistical description.

\section{Result}

We collected the data of nutritional status from Medical equipment testing, the domain include transferrin and albumin. Overall, every domain is changed to better status in the result. Between the control group and intervention group, they have different result of improvement as they receive different nursing services. In particular, the Albumin information shown intervention group have greater improvement after nursing that that of control group (from 59.24 \pm 5.07 to $49.16 \pm 2.01$ vs from $60.12 \pm 4.73$ to $57.72 \pm 2.33$ ).

Table 1. Nutritional Status [albumin $(g / L) \&$ transferrin $(g / L)]$.

\begin{tabular}{|c|c|c|c|c|c|c|c|c|}
\hline \multirow{2}{*}{ Projects } & \multicolumn{4}{|c|}{ Transferrin $(g / L)($ Mean \pm SD) } & \multicolumn{4}{|c|}{ Albumin (g/L) (Mean \pm SD) } \\
\hline & $\mathbf{B N}$ & FN & T-text & P Value & BN & FN & T-text & P Value \\
\hline Intervention Group $(\mathrm{n}=37)$ & $2.25 \pm 0.17$ & $1.61 \pm 0.20$ & 7.212 & $<0.005$ & $59.24 \pm 5.07$ & $49.16 \pm 2.01$ & 12.314 & 0.024 \\
\hline Control Group $(\mathrm{n}=37)$ & $2.12 \pm 0.26$ & $1.97 \pm 0.24$ & 4.510 & $<0.005$ & $60.12 \pm 4.73$ & $57.72 \pm 2.33$ & 4.448 & $<0.005$ \\
\hline T-text & 0.551 & 6.219 & - & - & 0.164 & 14.389 & - & - \\
\hline$P$ Value & 0.573 & 0.031 & - & - & 0.144 & $<0.005$ & - & - \\
\hline
\end{tabular}

$\mathrm{BN}=$ before the nursing

$\mathrm{FN}=$ after the nursing

In Tables 2, 3 domains of complications status have similar result in our research. In two different group, intervention group have less complications in the participants, the number is half as many as the control group ( 2 vs 4,3 vs 6,3 vs 7 ).

Table 2. Complications Status.

\begin{tabular}{llll}
\hline Projects & Upper gastrointestinal hemorrhage & Functional renal failure & Cirrhotic infection \\
\hline Control Group $(\mathrm{n}=37)$ & $4(10.8 \%)$ & $6(16.2 \%)$ & $7(18.9 \%)$ \\
Intervention Group $(\mathrm{n}=37)$ & $2(5.4 \%)$ & $3(8.1 \%)$ & $3(8.1 \%)$ \\
$\mathrm{X}^{2}$ & 5.210 & 0.047 & 3.326 \\
$\mathrm{P}$ Value & $<0.005$ & 0.738 & $<0.005$ \\
\hline
\end{tabular}

Table 3 shown the change of mental health in the treatment process. The participants finish the SDS and SAS questionnaires in before and after nursing so that they report the change of mental health. Base on mental health change, intervention group have greater change in anxiety status than

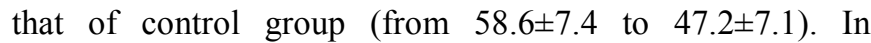
depression status, two groups have similar improvement in nursing service result.
Table 3. The score of anxiety and depression from SDS and SAS (Mean $\pm S D)$.

\begin{tabular}{llll}
\hline Projects & & SAS & SDS \\
\hline \multirow{2}{*}{ Intervention group $(\mathrm{n}=91)$} & $\mathrm{BN}$ & $58.6 \pm 7.4$ & $59.7 \pm 6.7$ \\
& FN & $47.2 \pm 7.1$ & $51.6 \pm 6.5$ \\
Control group $(\mathrm{n}=91)$ & $\mathrm{BN}$ & $62.5 \pm 7.4$ & $59.7 \pm 5.7$ \\
& FN & $56.3 \pm 7.2$ & $53.6 \pm 5.5$ \\
\hline $\mathrm{BN}=$ before the nursing & & & \\
$\mathrm{FN}=$ after the nursing & & &
\end{tabular}




\section{Discussion}

Liver cirrhosis is the final outcome of various liver disorders associated with hepatic encephalopathy, ascites, peritonitis and gastrointestinal bleeding [15]. Some researcher thinks the cirrhosis has become more prevalent in recent decades and it is a common cause of mortality all over the world [16]. As a result, when hepatic disorders occur, they are often accompanied by varying degrees of malnutrition [17]. Previous studies have shown that patients with liver cirrhosis generally suffer from varying degrees of malnutrition which is closely associated with poor quality of life and poor prognosis [18]. Furthermore, more attention has been paid to the nutritional status of patients with liver diseases. Malnutrition is a potentially modifiable condition, and it is very important to identify malnourished patients to ensure timely nutritional intervention. But the assessment methods is a challenge for nutritional status. Because decompensated patients with cirrhosis often experience varying degrees of edema and ascites as a result of portal hypertension and hypoproteinemia. Due to the presence of ascites and edema, these traditional anthropometric assessment methods cannot accurately assess nutritional status in patients with cirrhosis [19].

Based on the research result described above, the nutrition care service provides great improvement to the treatment result. However, nutrition care service does not have a good effect on partial results, such as transferrin, complications status and depression status. In nutritional status research, albumin index and transferrin index are reduced after nursing service, it indicated liver function of patient is continuing to recover. Compare with the data of control group and intervention group, albumin index change of two groups have great different. It show nutrition care service provide different result in result, nutrition care service provide better improvement result than that of control group. Additionally, complications status show all patients have few complications after treatment, the reason is that the simple size is limit the effective result so that the complications status result have not great different between control group and intervention group. In mental health of patient, all patients has severe anxiety and depression as liver cirrhosis and treatment bring pressure, pain and inconvenience to patient. The nutrition care service has good function to ease patient's anxiety in result. Our study also has some deficiencies to assess nutrition care service on patient with liver cirrhosis, that include the simple size limit the result of research and there were significant differences in postoperative behavior so that research result is effected in mental health.

\section{References}

[1] World Health Organization (WHO). Report Global hepatitis report 2017, Switzerland, (Geneva). 2017.

[2] Kumar A, Upadhyay V, Agrawal AK, Pandey P. N. A mathematical modeling of two phase hepatic mean blood flow in arterioles during liver cirrhosis. International Journal of Applied Research. 2017; 3 (7): 506e507.
[3] Marchesini G, Bianchi G, Amodio P, et al. Factors associated with poor health-related quality of life of patients with cirrhosis. Gastroenterology 2016; 120: 170-178.

[4] Martin LM, Sheridan MJ, Younossi ZM. The impact of liver disease on health-related quality of life: a review of the literature. Curr Gastroenterol Rep 2018; 4: 79-83.

[5] Yang JD, Kim WR, Coelho R, Mettler TA, Benson JT, Sanderson SO, et al. Cirrhosis is present in most patients with hepatitis $\mathrm{B}$ and hepatocellular carcinoma. Clinical Gastroenterology and Hepatology. 2014; 9 (1): 64e70.

[6] Bernardi M, Caraceni P. Novel perspectives in the management of decompensated cirrhosis. Nat Rev Gastroenterol Hepatol 2018; 15: 753e64.

[7] Swan WI, Vivanti A, Hakel-Smith NA, Hotson B, Orrevall Y, Trostler N, et al. Nutrition care process and model update: toward realizing people-centered care and outcomes management. J Acad Nutr Diet 2017; 117: 2003e14.

[8] Lacey K, Pritchett E. Nutrition Care Process and Model: ADA adopts road map to quality cape and outcomes management. J Am Diet Assoc 2016; 103: 1061e72.

[9] Daniel B, Alexandra K, Koen V. Process models in ditetic care: a comparison between models in Europe. Ernahrungs Umsch 2018; 65: 154e63.

[10] Kushner RF, Ayello EA, Beyer PL, et al. National Coordinating Committee for Nutrition Standards clinical indicators of nutrition care. J Am Diet Assoc. 1994; 94 (10): 1168-1177.

[11] National health expenditure data. Centers for Medicare \& Medicaid Services website. Accessed May 3, 2019.

[12] Desroches S, Lapointe A, Galibois I, Deschenes SM, Gagnon MP. Psychosocial factors and intention to use the nutrition care process among dietitians and dietetic interns. Can J Diet Pract Res 2018; 75. 48-48.

[13] White D, Leach C, Sims R, Atkinson M, Cottrell D. Validation of the Hospital Anxiety and Depression Scale for use with adolescents. Br J Psychiatry 1999; 175: 452-454.

[14] El-Rufaie O, Absood G. Validity study of the Hospital Anxiety and Depression Scale among a group of Saudi patients. Br J Psychiatry 1987; 151: 687-688.

[15] Schuppan D, Afdhal NH. Liver cirrhosis. Lancet. 2018; 371 (9615): 838-851.

[16] Moon AM, Singal AG, Tapper EB. Contemporary epidemiology of chronic liver disease and cirrhosis. Clin Gastroenterol Hepatol. 2019; (19) 30849-3.

[17] Tandon P, Raman M, Mourtzakis M, Merli M. A practical approach to nutritional screening and assessment in cirrhosis. Hepatology 2017; 65 (3): 1044e57.

[18] Maharshi S, Sharma BC, Srivastava S. Malnutrition in cirrhosis increases morbidity and mortality. J Gastroenterol Hepatol 2015; 30 (10): 1507e13.

[19] Plauth M, Cabre E, Riggio O, Assis-Camilo M, Pirlich M, Kondrup J, et al. ESPEN guidelines on enteral nutrition: liver disease. Clin Nutr (Edinburgh, Scotland) 2016; 25 (2): 285e94. 